\title{
LA TRANSICIÓN DEMOCRÁTICA Y LOS ACUERDOS EN EDUCACIÓN EN EL PERÚ
}

\author{
Manuel Iguiñiz Echeverría (*)
}

SÍNTESIS: La formulación de acuerdos de política educativa nacional en el Perú se produce como parte de un proceso político más amplio: la transición democrática que cambia el régimen autocrático de Fujimori.

El Acuerdo Nacional es el más importante proceso de renovación de la política en la transición democrática iniciada a finales del año 2000. Es una entidad de concertación de políticas de Estado, como respuesta a la imposición durante una década de un régimen autocrático y mafioso.

El Acuerdo Nacional establece 30 políticas de Estado. Incluye un objetivo exclusivamente dedicado a educación, auque otros también lo aludan. Así, la política educativa se ubica junto a otras en un pacto global de cambio del país.

En este artículo se recuperan antecedentes de América Latina y del Perú que favorecen llegar a acuerdos con base social y política amplia. En el caso peruano, además del Acuerdo Nacional, están recogidos la Consulta Nacional por la Educación, los programas del Ministerio de Educación, y el proceso democrático en la elaboración de la reciente Ley General de Educación.

En un reciente proceso de reactivación del Acuerdo Nacional, la educación ya no se presenta como la política de cambio social casi milagrosa, sino que se pasa a concretar de manera original ese objetivo en un denominado Pacto Social de Compromisos Recíprocos, que aparece como oportunidad de romper la inercia que domina el actuar educativo, y de movilizar actores importantes del país en torno a objetivos de corto plazo.

SÍNTESE: A formulação de acordos de política educacional nacional no Peru se produz como parte de um processo político mais amplo: a transição democrática que muda o regime autocrático de Fujimori.

O Acordo Nacional é o processo de renovação da política na transição democrática mais importante iniciada no final do ano 2000. É uma

*Ex viceministro de Educación, actualmente es asesor de la Alta Dirección del Ministerio de Educación, miembro de las asociaciones Foro Educativo y Tarea, y catedrático de Política Educativa Peruana en la Universidad Nacional Mayor de San Marcos, Perú. 
entidade de acordos de políticas de Estado, como resposta à imposição durante uma década de um regime autocrático e mafioso.

o Acordo Nacional estabelece 30 políticas de Estado. Inclui um objetivo exclusivamente dedicado à educação, ainda que outros também o aludam. Assim, a política educacional se coloca junto a outras em um pacto global de mudança do país.

Neste artigo, são recuperados antecedentes da América Latina e do Peru que favorecem chegar a acordos com ampla base social e política. No caso peruano, além do Acordo Nacional, estão recolhidos, à Consulta Nacional pela Educação, os programas do Ministério da Educação e o processo democrático na elaboração da recente Lei Geral de Educação.

Em um recente processo de reativação do Acordo Nacional, a educação já não se apresenta como a política de mudança social quase milagrosa, mas que passa a se concretizar de maneira original esse objetivo em um denominado Pacto Social de Compromissos Recíprocos, que aparece como oportunidade de romper a inércia que domina o atuar educativo, e de mobilizar atores importantes do país em torno dos objetivos de curto prazo.

\section{INTRODUCCIÓN}

La búsqueda de acuerdos nacionales en educación, una de las prácticas democráticas novedosas en la política educativa en el Perú, está marcada por la transición democrática iniciada a fines del 2000. Es decir, expresa reacciones ante situaciones limite de reciente data. La formulación de esas políticas es, sin embargo, tributaria de propuestas anteriores, tanto de la sociedad civil en el país, como de formulaciones de políticas en América Latina y el Caribe, planteadas como respuestas al incumplimiento de la promesa de la educación republicana frente a la sociedad.

\section{LOS ACUERDOS COMO BASE DE LAS POLÍTICAS DE ESTADO EN LA REGIÓN}

Una de las referencias para seguir cambiando la actual política educativa proviene del planeamiento de llegar a acuerdos nacionales en educación, surgido en la segunda mitad de la década de los 80 .

Los logros y flaquezas de la educación latinoamericana y caribeña merecieron balances críticos desde las esferas oficiales de la educación, con señalamiento directo de los condicionamientos contex- 
tuales. Así, en la Declaración de Guatemala ${ }^{1}$, los ministros de Educación argumentaron la necesidad de esos acuerdos nacionales, reconociendo factores como los que siguen:

- La imposibilidad de cumplir las metas educativas en América Latina y el Caribe. Se señala que, con los actuales ritmos de la acción educativa, los objetivos del Proyecto Principal no se podrían alcanzar ni en alfabetización ni en la «universalización de la escolaridad básica sin cambios sustanciales en la calidad» de este servicio. La mencionada reunión constata así el crónico desfase entre resoluciones y realizaciones, que más recientemente se repitió para el compromiso de Educación para Todos;

- La crisis económica está en la base de la crisis política, porque dificulta construir un destino común favoreciendo el fracaso escolar. Es clara la crítica sobre las políticas de desarrollo que no redistribuyen y que olvidan la identidad cultural, así como la participación social. «La desigualdad entre los países y los grupos sociales aumenta rápidamente, al límite de poner en peligro las posibilidades de construir un mundo donde cada ser humano y cada sociedad puedan aprovechar plenamente sus potencialidades y recursos»;

- Se critica el enfoque sectorial, ya que este enfoque tradicional olvida que las carencias educativas «no son productos aislados e independientes» de los factores sociales, políticos, culturales, ambientales y económicos. Asimismo, se hace la constatación de que las condiciones de pobreza neutralizan, en gran medida, los efectos de las acciones educativas. En ese marco, no es de extrañar el énfasis en estrategias integrales en todos los campos de la gestión, la pedagogía y la política educativa. Para los contextos de pobreza se requieren planes educativos de emergencia «que permitan movilizar recursos extraordinarios, a través de procedimientos excepcionales, adecuados a la gravedad de las situaciones que es preciso afrontar».

${ }^{1}$ Declaración de Guatemala (1989): Boletín 19, agosto, UNESCO-OREALC, Santiago de Chile. 
- Los propósitos de los ministros exigen la movilización no sólo de los presupuestos de educación, sino de todos los «recursos humanos y materiales disponibles en la sociedad, durante plazos prolongados».

No extraña en este contexto la demanda de que se formulen grandes consensos educativos nacionales en torno a los objetivos del Proyecto Principal.

Esta apuesta por los acuerdos nacionales tuvo vaivenes en la región en la década de los 90; por ejemplo, en la reunión de ministros en Santiago, en 1993, se puso énfasis en la gestión de un modo que obnubiló innecesariamente las dimensiones política, social y pedagógica. La propia O ficina Regional de la UNESCO reconoce esos vaivenes, y considera que en la reunión de ministros en Kingston, en 1996, «vuelven a aparecer énfasis que fueron prioridades hace una década. Se recupera con mayor fuerza el significado ético de la educación para asegurar una mayor equidad y una construcción de la identidad cultural. Se enfatiza la necesidad de que las políticas educativas incluyan a los excluidos, no tanto a través de medidas compensatorias como ocurría en el pasado, sino introduciendo modificaciones en el sistema educativo común que permitan ajustar la enseñanza a las diferencias individuales, sociales y culturales. Se vuelve a destacar la importancia de fortalecer la función del Estado para asegurar la igualdad de oportunidades, que no había estado presente desde la reunión de Bogotá de 1987»².

\subsection{GOBERNABILIDAD DE LOS SISTEMAS EDUCATIVOS}

De la búsqueda de acuerdos para la realización de los programas acordados se pasa a plantear una mirada más compleja a la crisis de los sistemas educativos. Crisis de legitimidad y respaldo social. Fue así como se propuso analizar la viabilidad de contar con una verdadera política. En la Cumbre Iberoamericana de 1996 y en la reunión previa de ministros de Educación en Concepción ${ }^{3}$, se amplió el marco de la cuestión a facetas diferentes como la gobernabilidad democrática en la región, y, dentro de ella, la gobernabilidad de los sistemas educativos.

2 UNESCO-OREALC (1998): «La UNESCO y el desarrollo educativo en América Latina y el Caribe», en Boletín 45, p. 16, abril, Santiago de Chile.

3 «Gobernabilidad democrática y gobernabilidad de los sistemas educativos», Declaración de Concepción, en Revista Iberoamericana de Educación, núm. 12, oEI, 1996. 
El sistema político democrático ya no es sólo un logro consensual; su relevancia para la ciudadanía es un asunto de varias aristas. Reconocer las brechas sociales y las exclusiones es un referente básico, que estuvo acentuado en los acuerdos de los ministros de la región a fines de la década de los 80 , y se retoma en las consideraciones y propuestas sobre la gobernabilidad a mediados de los 90 , en Concepción, marcando una diferencia con el extendido supuesto de que no existe una causa económica de la crisis de gobernabilidad política y en el decaimiento educativo, pues el modelo marcha y sólo falta la reforma del Estado

A la vez, uno de los énfasis en la reflexión de la declaración de los ministros en Concepción fue el de la representación política y su crisis como clave de la gobernabilidad. Esa crisis amerita como cuestión esencial «la recuperación de la política como dimensión constitutiva del ser humano en sociedad, como actividad noble y necesaria; en ese sentido, el servicio público y la función pública deben recuperar prestigio, nobleza, respeto».

Si bien la educación es tributaria de las condiciones de la política nacional, es también factor activo en la democratización social; de ahí que en la declaración de Concepción se recapitulen formas en las que se puede concretar la contribución de la educación a la gobernabilidad democrática.

\section{LOS AVATARES DE LA DEMOCRACIA PERUANA Y EL ACUERDO EN EDUCACIÓN}

\subsection{EL HASTÍO ANTE LA INESTABILIDAD DE LA POLITICA EDUCATIVA}

La formulación de una voluntad política de acuerdo nacional en educación no acompañó en el Perú, como sí en algunos países, las resoluciones de los ministros y la Declaración Educación para Todos. Las fuerzas políticas no la incorporaron a su repertorio sino años después, siendo el gobierno de Fujimori, en la década de los 90, la antítesis de los consensos.

Sin embargo, no dejaron de oírse voces que ya en períodos anteriores fueran incitadoras de ese tipo de acuerdos. En 1958 el historiador Jorge Basadre, a la sazón ministro de Educación, ya planteaba el reconocimiento de una notable expansión del sistema con deficiencias 
de calidad, Asimismo, en la primera mitad de la década de los 70, la Reforma Educativa del gobierno militar criticó la pedagogía tradicional, el déficit de calidad, el elitismo y la expresión de una cultura inauténtica, producida sobre todo por la histórica dominación externa del país. Finalmente, en el primer gabinete de Fujimori, la gestión educativa del Ministerio de Educación preparó la convocatoria de un debate nacional que diera lugar a una política educativa de consenso, que no llegó a hacerse pública.

Con el autogolpe del 2002 el régimen de Fujimori se configuró como una de las dictaduras más ingeniosas en montar un sistema de dominación, con una democracia insincera, con mecanismos de legitimación, con el uso intensivo del servicio de inteligencia, con el empleo de los medios de comunicación masiva nublando la percepción de su carácter de régimen mafioso. A su vez, en este período se organizaron sectores de educadores alrededor de fórmulas de un proyecto educativo nacional. Al producirse la remoción del primer gabinete de Fujimori, en 1990, una parte del grupo que había preparado el programa del Ministerio de Educación, con la incorporación de otras personas, conformó la asociación Foro Educativo, de inédita pluralidad política y diversidad profesional, como espacio de propuestas desde la sociedad civil que enfrentara la inestabilidad crónica de la política educativa. Esta asociación planteó la búsqueda de acuerdos y su expresión en políticas de Estado, organizados en un proyecto educativo nacional que se venía reiterando en diversos eventos de educación.

\subsection{LA EDUCACIÓN EN EL ACUERDO DE GOBERNABILIDAD}

La política partidaria incorporó recientemente, en su acervo de recursos, el pacto político entre fuerzas diversas, más abarcador que las alianzas electorales y los bloques para gobernar. El juego político se dotó de un espacio de contacto y de acuerdo diferente al Parlamento. En 1999, 14 partidos y organizaciones políticas suscribieron el Acuerdo de Gobernabilidad. Fue el inicio de un tardío cambio en la manera de hacer política. El Acuerdo, desde entonces, ha estado presente con intermitencia en la escena pública. Ante la cercanía de las elecciones del año 2000, el Acuerdo propuso cuatro orientaciones: el apoyo al gobierno democrático de unidad nacional surgido de las elecciones para restituir el Estado de Derecho; una política económica diferente; la reforma del Estado y la descentralización; y una política social para la igualdad de oportunidades 
y para la disminución de la pobreza. En relación con la política social en educación se indica:

1. El Estado garantizará la gratuidad de la enseñanza pública en todos los niveles, asegurando que nadie quede sin acceso a la enseñanza y la capacitación, como resultado de su falta de recursos económicos. Esto supone una mejora sustancial de la calidad de la educación que otorga el Estado, la promoción y el desarrollo de la escuela pública como vehículo de conocimiento, integración social y participación de la sociedad, así como el compromiso de mejorar la profesionalización, la calidad y las remuneraciones del magisterio ${ }^{4}$.

Si bien el acuerdo de los partidos políticos no significó ser el factor determinante de la caída del régimen autocrático de Fujimori, esa concertación fue un elemento de la transición para recuperar el Congreso de la Republica y el funcionamiento plural de la Mesa de Diálogo, con asistencia de la OEA, así como para la instalación del gobierno de transición.

\section{CAMBIO DE RÉGIMEN POLÍTICO}

Algunos aportes fundamentales del gobierno de transición en el cambio de régimen fueron los siguientes: restablecer el principio de legalidad; reconstituir la alternancia en el gobierno y la división de poderes; y la ampliación del lugar de la sociedad civil en las políticas públicas. Im portantes decisiones fueron em prender la lucha contra la comupción del régim en anterior, y la constitución de la Com isión de la Verdad para elaborar un inform e a la sociedad peruana sobre dos décadas de violencia política.

\subsection{EDUCACIÓN Y SOCIEDAD}

Los sentimientos de rechazo a la corrupción hacen notorio el olvido de que el cambio educativo está ligado a la experiencia social. Es lo que asumió el presidente del Consejo de Ministros, Pérez de Cuéllar, pues en su programa insiste en que la tarea de la educación ética y cívica requiere de la escuela y del Ministerio de Educación, pero pide «para ello

${ }^{4}$ Publicado en el diario La República, 26 de noviembre de 1999. 
una especial cooperación a los medios de comunicación, a las organizaciones de la sociedad civil, a las asociaciones de padres de familia y a las propias organizaciones juveniles». De manera acertada se vincula la democratización educativa con su calidad y con su expresión en logros básicos para todos, lo que implica superar el esquema sectorial, y así «contar con acciones específicas en los campos de la pobreza, la interculturalidad, el multilingüismo y la dispersión de la población rural».

La educación se expresa como un punto importante en los acuerdos nacionales y locales para el desarrollo humano. Se llevaron a cabo diversos espacios de concertación a escala regional y local, como los obtenidos en las nuevas Mesas de Lucha Contra la Pobreza ${ }^{5}$.

\subsection{EL ACUERDO NACIONAL}

En el gobierno de Alejandro Toledo, en julio de 2002, se suscribe el Acuerdo Nacional, que marca un cambio muy importante en la política peruana, pues lo integran los partidos políticos junto con importantes instituciones de la sociedad civil como iglesias, organizaciones sindicales, empresarios, figuras de la cultura, el Congreso Nacional y el Poder Ejecutivo ${ }^{6}$. El Acuerdo expresó la reacción ante la crisis política, la búsqueda de rumbos y la voluntad de formular un proyecto nacional.

El Acuerdo formuló políticas de Estado, abordando temas cruciales puestos en el foco por la transición democrática, así como viejos problemas estructurales del país. Está conformado por 30 políticas de Estado, que tienen que ver con cuatro objetivos básicos: dem ocracia y Estado de Derecho; equidad y justicia social; com petilividad delpaís; y Estado eficiente, transparente y descentralizado.

La ubicación de la educación com o com ponente de un programa global, tal como se planteó en 1999, se amplía en el Acuerdo

${ }^{5}$ Carta Social. Compromiso por el desarrollo y la superación de la pobreza, junio 2001. Por su parte, la v CONADES tuvo como resultado las Propuestas para la transición democrática, 2001. El Ministerio de la Presidencia publicó en 2002 los Planes Concertados de Desarrollo Departamental.

${ }^{6}$ Rafael Roncagliolo: «Pacto Nacional y Pacto Social», diario La República, 15 de abril de 2004. El autor, secretario técnico del Acuerdo Nacional, señala que en la frecuente comparación del Acuerdo Nacional con los Pactos de la Moncloa, no se repara en varias diferencias, una de las cuales es la presencia directa de las organizaciones de la sociedad civil. 
Nacional. La educación es una de las políticas de equidad y de justicia (ver anexo de la Política en Educación del Acuerdo Nacional). Ubicar la educación dentro de un amplio abanico de políticas, y a la vez con importantes funciones sociales propias, es un logro que recoge vertientes de pensamiento más integradoras. Al reconocer la centralidad de la educación no se pretende nublar la densidad consustancial a todos los ámbitos del desarrollo humano, y se supera la pretensión de desvincular la educación como clave independiente de dicho desarrollo, sin requerimientos de cambios en otros ámbitos como los económicos.

La política propuesta recoge también el momento de la transición, con su alusión a la ética y a los valores, así como a la ciudadanía, y la referencia a la escuela publica. Asume igualmente los temas de las estructuras de desigualdad históricas, las brechas, la prioridad a la infancia, y las exigencias de calidad y de interculturalidad.

\subsection{LA CONSTRUCCIÓN DEMOCRÁTICA DE LA POLÍTICA EDUCATIVA}

Si la transición modificó el ritmo de la política general en el país, fue muy acertado que el primer punto del programa presentado por el nuevo gobierno en materia de educación fuera la elaboración democrática de la política educativa. «La tarea principal en el tema educativo será convocar a la construcción democrática de las propuestas que debieran orientar el esfuerzo nacional, con independencia de los cambios de gobierno, en los próximos años».

Así se emprendió el cambio hacia un régimen democrático, abriendo las deliberaciones para la formulación de políticas públicas en educación. Entre los elementos con valor propio y de potencialidad transformadora que se incorporaron a las políticas educativas podemos enumerar los siguientes: el Ministerio de Educación, en el gobierno de transición, recogió propuestas de entidades externas, como el Foro Educativo $^{7}$, para la realización de la Consulta Nacional por la Educación; el gobierno abrió espacio a la crítica respecto de la situación educativa, empezando por autorizar la publicación de la medición de aprendizaje de

\footnotetext{
7 Destacan entre los aportes del Foro Educativo para la Consulta Nacional y para las políticas nacionales: Propuesta de Foro Educativo a un acuerdo nacional para el 2001-2005, abril 2001. Vote por la educación, Agenda de prioridades 2000-2005, marzo 2000. Por una educación de calidad para todos. Compromisos asumidos por el Perú hasta el 2015, julio 2001. Entre las instituciones que individualmente formularon propuestas globalesestánIPAE (CADE 2001), Tarea.
} 
UNESCO, que evidencia que la escuela mayoritaria no logra la satisfacción de las necesidades básicas de aprendizaje; el Ministerio de Educación dio pasos iniciales en programas de renovación pedagógica y de democratización de la gestión escolar para enfrentar las brechas en el aprendizaje, que incluyeron el relanzamiento de la educación inicial, la educación de jóvenes y adultos ${ }^{8}$, y la educación bilingüe intercultural. También se inició, de manera presurosa, una renovación curricular de conjunto de la educación secundaria, y se retomó la idea de impulsar la educación rural, clave para la política de equidad en la mayoría de las nuevas regiones. Asimismo, el programa para la renovación pedagógica, aprovechando las nuevas tecnologías. Se recuperó la idea de la revalorización de la función pública y de la profesionalización de la gestión. Se reinstaló la Oficina de Desarrollo Magisterial, y se abordaron los temas de la autonomía y de la democratización escolar.

La Consulta Nacional por la Educación significó un importante paso en la generación de políticas educativas. Dirigida por una comisión independiente, se orientó a recoger opiniones de distintos sectores del país.

Propuso los siguientes objetivos para la educación:

- Una educación que prepare para ser ciudadanos incorporados plenamente a la sociedad.

- Dar a la profesión docente su justo valor.

- La educación es tarea de todos.

- Descentralizar y democratizar la educación.

- Brindar a todos el acceso a una educación de calidad.

- Todos los peruanos tenemos derecho a conocer y a evaluar los avances de la educación.

- Invertir más y mejor en educación.

- Llevar la educación superior a estándares de calidad internacional.

8/ Conferencia Nacional, Educación de Jóvenes y Adultos en el Perú, 2001, Ministerio de Educación. 
Pero hay que resaltar que el informe hizo la conexión entre la educación y el marco de crisis del país que habitualmente se extraña en las políticas educativas. Sin embargo, como propuesta de ancha base no recoge unanimidad de criterios. Así, las propuestas de mercado y de competencia entre escuelas no encuentran en estas iniciativas o en el Acuerdo Nacional un asidero. Hay que recordar que fueron sugerencias de Fujimori que pronto las retiró él mismo, tras recibir criticas desde varios sectores. No obstante lo novedoso de la Consulta Nacional, su informe tuvo insuficiente difusión y aprovechamiento porque no se superó la tradición de prescindencia de propuestas previas.

Posteriormente, durante el gobierno electo, el Ministerio de Educación, después de muchos años, hizo pública una propuesta de política educativa global, los Lineamientos de política 2001-2006. Estos lineamientos coinciden con la Consulta Nacional por la Educación en retomar la función de la educación en cuanto a formar ciudadanos con capacidades de construir bienestar, y revaloran el significado democrático de la educación pública. Asimismo, consideran como aspecto central un pacto social y político, y potencian programas pedagógicos de equidad y calidad para enfrentar las brechas existentes en el aprendizaje de millones de estudiantes.

Se dio continuidad a normas del gobierno de transición en gestión escolar; se ampliaron los criterios de evaluación del concurso para el ingreso de los docentes a la carrera pública, pues se incluyó la participación de la institución educativa en el proceso y se realizó una prueba nacional de aprendizajes. Se concretó otra antigua demanda de sectores de educadores conformando el Consejo Nacional de Educación.

\subsection{LA DESCENTRALIZACIÓN EDUCATIVA}

La primera característica del reinicio de la descentralización, que el autogolpe de Fujimori había abortado una década atrás, consiste en que forma parte de un cambio de régimen político y no de un cambio de gobierno. Es decir, predominan razones políticas por encima de las lógicas, como por ejemplo de ajuste fiscal, que ya fuera realizado de manera durísima a inicios de los 90.

La descentralización puesta en valor por el hipercentralismo de Fujimori tuvo respaldo de los partidos en las campañas electorales de los años 2000 y 2001 , y, luego, el Congreso Nacional asumió el protagonismo 
con modificaciones a la Constitución y con la promulgación de una legislación importante.

Para algunos, el momento de empezar la descentralización fue inadecuado. Por nuestra parte pensamos que, por el contrario, fue el adecuado, porque se convirtió en uno de los impulsores de la democratización, pero se inició con algunos problemas serios, como la herencia de la conversión de los actuales departamentos en regiones.

Si con el proceso democrático se han ampliado los espacios de participación de la sociedad civil en los asuntos públicos, ahora, con la descentralización, se afianzan legislativamente algunos en los niveles local y regional. La legislación es más clara en las características deseables del Ministerio, en las de las regiones y las escuelas, pero lo es menos en el nivel local en cuanto a las funciones de la municipalidad, una de las cuestiones a definir para el diseño más preciso de la descentralización.

\subsection{LEY GENERAL DE EDUCACIÓN}

El Congreso Nacional tuvo como iniciativa principal en educación la elaboración de la Ley General de Educación, una buena decisión política llevada a cabo a través de la legislación. Se discutió mediante una consulta democrática, y llegó a concitar el acuerdo en el Congreso. Se trata de un pacto con contenidos políticos democráticos, con amplia valoración de la participación de la sociedad civil y con un rol activo del Estado. Reafirma el derecho a una educación de calidad para la igualdad de oportunidades. Está actualizada en materia pedagógica, y recoge aportes de varias vertientes del pensamiento educativo y político. Así, se afirma la centralidad del educando; la educación entendida como proceso durante toda la vida y en ámbitos sociales diferentes; la evaluación del sistema, de los aprendizajes y de los docentes; y la acreditación de instituciones educativas. Busca comprometer a los actores para su implicación responsable, y la acción intersectorial del Estado en sus distintos niveles de gobierno. 


\subsection{UNA COYUNTURA DEMANDANTE}

Si la lucha contra la autocracia y el inicio de la transición llevó al acuerdo político nacional, también las demandas del sector educativo de corto plazo reclaman nuevos acuerdos.

\section{Reclamo de redistribución}

Mientras el crecimiento no se traduzca en redistribución, esta exigencia no se puede congelar en democracia, donde la demanda de varios sectores está más presente.

El incremento del presupuesto no ha expresado una prioridad en la educación, sólo un ligero crecimiento. Por ello, no se llegará a alcanzar el más mencionado de los puntos del Acuerdo Nacional en educación de invertir un $6 \%$ del PBI en ella, y que es, a la vez, un pacto propuesto por la Declaración de Concepción. También se recuerda la promesa presidencial de duplicar el sueldo de los docentes con el 2006 como meta. No obstante, si bien en lo que va transcurrido de democracia se ha producido un significativo incremento de sueldos, éste es menor al ofrecido.

\section{La huelga de docentes y la declaratoria de emergencia}

El derecho a la huelga y el derecho a la negociación colectiva del sindicato se recuperaron después de diez años. Sin embargo, no se supo evitar una importante huelga magisterial que trastornó no sólo el escenario educativo, sino el político nacional ${ }^{9}$. Condujo a la declaración del estado de emergencia y obligó al debate de nuevos acuerdos sociales, entre los que destacaron dos: el de la carga tributaria, y el de la enorme diferencia en los sueldos del sector público, que aún no se termina de resolver.

La discontinuidad habida en la política del Ministerio de Educación en las cuestiones programáticas que se venían avanzando, y la desconexión con la comunidad educativa, afectan el manejo productivo de estos conflictos.

55, agosto, Lima.

${ }^{9}$ Manuel Iguiñiz (2003): «Señora huelga, señora ley», en Revista Tarea, núm. 
La difusión de los deficientes resultados de la prueba internacional PISA el año 2003 entre educandos de 15 años, agudiza la conciencia de la crisis educativa con sus bajos niveles de aprendizaje. Eso, en las postrimerías de la huelga, llevó al anuncio de una puesta en emergencia de la educación. No obstante, la declaración de emergencia no condujo a movilizar nuevos recursos ni a medidas excepcionales que reclam aron los $m$ in istros en Guatem ala. Es después de casiun año que elactualplan delm inisterio trata de dar form a a esa declaración.

\section{5. ¿PACTO FUNDACIONAL?}

La transición con todos sus límites, y a partir de sus mejores impulsos democráticos, ha dejado una nueva plataforma de posibilidades a la política educativa. Los procesos de cambio en el discurso de la política y en la formulación de lineamientos estratégicos, la importante legislación, así como los pasos iniciales de realización en la calidad de la educación, no se profundizarán de no mediar nuevos acuerdos en varios niveles y con actores multiplicados.

La creación de condiciones como sociedad y Estado están aún distantes para dar nacimiento a un pacto fundacional como el que se deriva de la relectura de nuestro pasado hecha en el Informe de la Comisión de la Verdad y Reconciliación ${ }^{10}$. Ese pacto es indispensable para posibilitar procesos de profundización democrática, con pluralidad cultural y con creciente equidad social en un marco de desarrollo productivo. Los miembros del Acuerdo Nacional y los actores de la política educativa tienen el encargo de ensanchar la democracia y la inclusión social.

\subsection{AVANZAR EN PROYECTO ACUERDOS Y PLANIFICACIÓN}

Los acuerdos y alianzas no han continuado nutriéndose con novedades de transformación de las fuerzas políticas ni de expresiones suficientes de solidaridad nacional para reformar con profundidad la educación. En el Congreso no se pudo culminar la reforma constitucional

\footnotetext{
${ }^{10}$ Carlos Iván Degregori (2004): «Desigualdades persistentes y construcción de un país de ciudadanos», en Cuestión de Estado, núm. 33-34, marzo, Instituto de Diálogo y Propuestas, Lima.
} 
que incorporara lo más democrático de la transición, a excepción del capitulo aprobado para iniciar la descentralización.

En un marco de incertidumbre política y de una economía que crece poco y que redistribuye menos, nuevos acuerdos específicos entre más actores son indispensables, como lo es el fortalecimiento de las organizaciones de la sociedad civil. Hay espacios y oportunidades no aprovechados por la Consulta Nacional por la Educación, como los logros de definición de propósitos, los lineamientos de políticas y de prioridades de acción, así como los que pueden brindar entidades de participación y de vigilancia. Sin embargo, la coyuntura ofrece una nueva oportunidad de avanzar en realizaciones educativas y en la contribución de la comunidad educativa a la gobernabilidad democrática. Se trata del Pacto Social de Compromisos Recíprocos, elaborado recientemente por el Consejo Nacional de Educación por encargo del Acuerdo Nacional, que abre posibilidades para relanzar iniciativas y para lograr a corto plazo compromisos específicos y articulaciones entre importantes instituciones públicas y privadas.

Ese encargo es posible por el relanzamiento del Acuerdo Nacional, que es uno de los resultados más alentadores de reacción ante la incertidumbre política. De los firmantes iniciales del Acuerdo, sólo la conservadora Unidad Nacional se mantiene al margen de este relanzamiento.

El Proyecto Educativo Nacional es otro estimulo para pactos más específicos. El debate sobre el Proyecto Educativo Nacional iniciado por el Consejo Nacional de Educación, puede confluir con el proceso del Foro de Educación para Todos para el Plan de Acción Nacional de seguimiento de los acuerdos de Dakar, y dar un paso importante para dotar a la educación de una planificación con sustento social. Con ello, servir a la elaboración de los planes educativos regionales que fortalezcan a la región como instancia de política y de planificación, lo cual, a su vez, exige una calificación de funcionarios regionales. Pero, sobre todo, requiere nuevos acuerdos con las regiones para evitar que la autonomía regional y la escolar se conviertan en administración autárquica de la pobreza, y en un localismo que traba la complementariedad entre los niveles de gobierno del país.

La nueva oportunidad de unir el plan de emergencia y el pacto social. La propuesta del Pacto Social de Compromisos Recíprocos, presentado por el Consejo Nacional de Educación, no sólo concreta el 
Acuerdo Nacional, sino que impulsa un cambio de intensidad y de ritmo de la política pública a corto plazo. La emergencia en educación afirmada por el plan del Ministerio de Educación, puede ser fructífera si se avanza al mismo tiempo en el pacto social de compromisos recíprocos, que también la incorpora. Así, se da una oportunidad valiosa de dar al programa de educación sustento y viabilidad, por la alianza de los principales actores de la política nacional. Su abordaje en serio ofrece una importante posibilidad de movilizar y de concertar un proceso de planificación, que incluya actores claves como el Ministerio de Educación, el Ministerio de Economía y Finanzas y otros ministerios, el Congreso Nacional, los sindicatos, los empresarios presentes en el Acuerdo Nacional, así como el Consejo Nacional de Educación, el Foro Educativo y las mesas regionales en educación. Con ello se podrá intentar convertir la educación en un tema de la agenda política, constituyéndose también en un aporte a la gobernabilidad democrática.

La gobernabilidad del sistema educativo. La Ley General de Educación, los avances en la discusión del Proyecto Educativo Nacional, y el inicio de la descentralización en el marco de la crónica discontinuidad de la conducción del sector educación, ameritan cambiar el modo de administrar la educación y avanzar hacia un gobierno no exclusivo del Ministerio, fortaleciendo la participación del Consejo Nacional de Educación y de un consejo de concertación con los directores regionales de Educación, como partes involucradas de manera obligatoria en las principales decisiones. Variar el sistema de dirección nacional y ganar continuidad, son requisitos necesarios para reconstituir una carrera pública en educación, cuya práctica desaparición dificulta enfrentar las resistencias burocráticas al cambio y a la moralización.

El desarrollo de una cultura de la participación y de vigilancia. Las mayores oportunidades y canales de participación legislados no aseguran la transformación duradera de la manera de entender la política y la educación ciudadana. Se requiere enfrentar el clientelismo en la educación por parte de los partidos, ya que está inscrito en la cultura política de manera extendida y arraigada. La experiencia de estos años confirma cómo la existencia de diversos núcleos de iniciativa política favorecen la elaboración de buenas políticas educativas. Asimismo, los favorecen el fortalecimiento de la capacidad critica de la sociedad civil y la vigilancia con implicación responsable. La mayor presencia de las organizaciones de participación y de vigilancia es uno de los componentes esenciales para exigir la profesionalización y la moralización en el sistema educativo público. 


\section{BIBLIOGRAFÍA}

ACUERDO DE GOBERNABILIDAD (1999): diario La República, 26 de noviembre.

BRASLAVSKY, C. (1989): «Evolución de los sistemas educativos en los procesos de transición a la democracia», en VV.AA: Educación en la transición a la democracia. Caos de Argentina, Brasil y Uruguay, UNESCO, Santiago de Chile.

DEGREGORI, C. I. (2004): «Desigualdades persistentes y construcción de un país de ciudadanos», en Cuestión de Estado, núm. 33-34, Lima, Instituto de Diálogo y Propuestas, marzo. Lima, agosto.

IGUIÑIZ, M. (2003): «Señora huelga señora Ley», en Revista Tarea núm. 55,

OEI (1996): «Declaración de Concepción». VI Conferencia Iberoamericana de Educación, en Revista Iberoamericana de Educación, núm. 12, septiembre-diciembre, Madrid, OEI.

— «Educación y gobernabilidad democrática» (monográfico), en Revista Iberoamericana de Educación, núm. 12, septiembre-diciembre, Madrid, OEI.

de abril.

RONCAGLIOLO, R.: «Pacto Nacional y pacto social», en diario La Republica, 15

TERCERA ReUnión DE MinISTROS DEL PROYECTO PRINCIPAL. DECLARACIÓN DE GUATEMALA (1989): Boletín 19, Santiago de Chile, UNESCO-OREALC, agosto.

UNESCO-OREALC (1998): "La UNESCO y el desarrollo educativo en América Latina y el Caribe», en Boletín 45, Santiago de Chile, abril.

VV.AA. (1995): ¿Es posible concertar las políticas educativas? La concertación de políticas educativas en Argentina y América Latina, Buenos Aires, Miño y Dávila Editores. 


\title{
ANEXO \\ DÉCIMO SEGUNDA POLÍTICA DE ESTADO
}

\author{
«ACCESO UNIVERSAL A UNA EDUCACIÓN PÚBLICA GRATUITA Y DE CALIDAD, \\ Y PROMOCIÓN Y DEFENSA DE LA CULTURA Y DEL DEPORTE»
}

Nos comprometemos a garantizar el acceso universal e irrestricto a una educación integral, pública, gratuita y de calidad que promueva la equidad entre hombres y mujeres, afiance los valores democráticos y prepare ciudadanos y ciudadanas para su incorporación activa a la vida social. Reconoceremos la autonomía en la gestión de cada escuela, en el marco de un modelo educativo nacional y descentralizado, inclusivo y de salidas múltiples. La educación peruana pondrá énfasis en valores éticos, sociales y culturales, en el desarrollo de una conciencia ecológica y en la incorporación de las personas con discapacidad.

Con ese objetivo el Estado:

a) Garantizará el acceso universal a una educación inicial que asegure un desarrollo integral de la salud, nutrición y estimulación temprana adecuada a los niños y niñas de cero a cinco años, atendiendo la diversidad étnico-cultural y sociolingüística del país.

b) Eliminará las brechas de calidad entre la educación pública y la privada, así como entre la educación rural y la urbana, para fomentar la equidad en el acceso a oportunidades.

c) Promoverá el fortalecimiento y la revaloración de la carrera magisterial, mediante un pacto social que devenga en compromisos recíprocos que garanticen una óptima formación profesional, promuevan la capacitación activa al magisterio y aseguren la adecuada dotación de recursos para ello.

d) Afianzará la educación básica de calidad, relevante y adecuada para niños, niñas, púberes y adolescentes, respetando la libertad de opinión y de credo. 
e) Profundizará la educación científica y ampliará el uso de nuevas tecnologías.

f) Mejorará la calidad de la educación superior pública, universitaria y no universitaria, así como una educación técnica adecuada a nuestra realidad.

g) Creará los mecanismos de certificación y calificación que aumenten las exigencias para la institucionalización de la educación pública o privada, y que garanticen el derecho de los estudiantes.

h) Erradicará todas las formas de analfabetismo invirtiendo en el diseño de políticas que atiendan las realidades urbanomarginal y rural.

i) Garantizará recursos para la reforma educativa, otorgando un incremento mínimo anual en el presupuesto del sector educación equivalente al $0.25 \%$ del $\mathrm{PBI}$, hasta que éste alcance un monto global equivalente al $6 \%$ del PBI.

j) Restablecerá la educación física y artística en las escuelas, y promoverá el deporte desde la niñez.

k) Fomentará una cultura de evaluación y vigilancia social de la educación, con participación de la comunidad.

I) Promoverá la educación de jóvenes y adultos y la educación laboral en función de las necesidades del país.

m) Fomentará una cultura de prevención de la drogadicción, pandillaje y violencia juvenil en las escuelas.

n) Fomentará y afianzará la educación bilingüe en un contexto intercultural.

Lima, Perú, 22 de julio de 2002 


\title{
Contactar
}

Revista lberoamericana de Educación

\author{
Principal OEI
}

